\title{
Using Feedback to Mitigate Coordination and Threshold Problems in Iterative Combinatorial Auctions
}

\author{
Bart Vangerven • Dries R. Goossens • Frits C. R. Spieksma
}

Received: 2 June 2019/Accepted: 14 February 2020/Published online: 31 March 2020

(C) The Author(s) 2020

\begin{abstract}
Package bids, i.e., bids on sets of items, are an essential aspect of combinatorial auctions. They can allow bidders to accurately express their preferences. However, bidders on packages consisting of few items are often unable to outbid provisionally winning bids on large packages. To resolve this, both coordination as well as cooperation are needed. Coordination, since smaller bidders need to bid on packages that are disjoint; cooperation, since typically bid increases from more than one bidder are required to overcome the threshold to outbid a larger package bid. The authors design an information system that supports the implementation of an iterative combinatorial auction; this system is specifically aimed at helping bidders overcome coordination and threshold problems. They study the effect of information feedback on the behavior of bidders in different auction settings. The authors test this in
\end{abstract}

Accepted after one revision by Natalia Kliewer.

Electronic supplementary material The online version of this article (https://doi.org/10.1007/s12599-020-00638-z) contains supplementary material, which is available to authorized users.

B. Vangerven $(\square)$

Schumpeter School of Business and Economics, University of Wuppertal, Gaußstraße 20, 42219 Wuppertal, Germany

e-mail: vangerven@uni-wuppertal.de

\section{R. Goossens}

Faculty of Economics and Business Administration, Ghent

University, Tweekerkenstraat 2, 9000 Ghent, Belgium

e-mail: dries.goossens@ugent.be

F. C. R. Spieksma

Department of Mathematics and Computer Science, Eindhoven

University of Technology, 5600 MB Eindhoven, The

Netherlands

e-mail: f.c.r.spieksma@tue.nl an experimental setting using human bidders, varying feedback from very basic information about provisionally winning bids/prices, to providing more advanced concepts such as winning and deadness levels, and coalitional feedback. The experiment indicates that coalitional feedback has a positive impact on economic efficiency in cases where difficult threshold problems arise; however, it appears to have an adverse effect when threshold problems are easy.

Keywords Auctions/bidding - Coordination and threshold problems · Bidder support - Laboratory experiment

\section{Introduction}

Combinatorial auctions (CAs) are allocation mechanisms that enable selling and buying multiple (indivisible) items simultaneously. In fact, CAs allow bidders to bid on sets of items (packages) and the auctioneer can allocate any package only in its entirety to the corresponding bidder. CAs have established themselves as a viable allocation mechanism in settings where market prices are not readily available, and bidders have sub- or super-additive valuations. CAs offer the possibility for a coalition of bids on small packages to jointly outbid a single bidder's claim on the complete set of items. However, two hurdles need to be overcome before a coalition can become winning.

(1) The coordination problem Bidders need to coordinate their bids and bid on complementary (i.e., nonoverlapping) sets of items. The coordination challenge lies in bidders having to discover such a set of individually profitable and collectively complementary packages, given that the number of possible 
packages rises exponentially with the number of items. This is complicated by the existence of cognitive limits on the number of packages people can concentrate on during the auction. For instance, experimental research by Scheffel et al. (2012) indicates that bidders only bid on 6 to 10 different packages, independent of the auction format, although the bidders had a multitude of packages with positive valuations to choose from. Coordination is hindered by the assumption that a bidder only knows his/her private valuation for these packages, and not the preferences of other bidders. In fact, in order to mitigate collusion, it makes sense to restrict communication between bidders (see e.g., Cramton and Schwartz 2000).

(2) The threshold problem Even if the coordination problem is overcome, the task of determining appropriate bid prices to displace the currently winning bid still remains. A complicating factor is that each bidder in a coalition has an incentive not to increase his/her bid. Indeed, the forgone revenue from unilaterally increasing one's bid falls entirely on the cooperating bidder, whereas the benefits extend to the non-cooperating bidders as well. Thus, the problem of choosing an appropriate bid price is non-trivial.

As far as we are aware, the term "threshold problem" was coined by Rothkopf et al. (1998), although the problem itself was mentioned already in e.g., Banks et al. (1989). Several papers on CAs discuss the threshold problem (e.g., Rothkopf et al. 1998; Day and Raghavan 2008; Brunner et al. 2010; Scheffel et al. 2012; Chernomaz and Levin 2012), however, in some cases the term "threshold problem" is used as a container concept for both the coordination and the threshold problem. Bichler et al. (2017) point out that the coordination problem has largely been ignored in the game-theoretical literature on CAs.

Both the coordination problem and the threshold problem are solved in the well-known Vickrey-Clarke-Groves (VCG) auction. Indeed, in the VCG auction, it is a weakly dominant strategy for bidders to report their valuations truthfully, which takes care of the threshold problem, and hence to bid on all packages for which they have a positive valuation, which deals with the coordination problem. However, the VCG auction is rarely used in practice due to a number of issues, such as very low auction revenues (see Ausubel and Milgrom 2006). The VCG outcome is also not necessarily a competitive equilibrium. Furthermore, there is still the issue of cognitive limits, making it unrealistic that bidders would effectively bid on each package they value.
Even though the coordination and threshold problem are also relevant in single round CAs, this paper focuses on iterative, ascending CAs. An iterative auction consists of multiple rounds, such that bidders can repeatedly increase their bids and/or introduce new bids. Our paper discusses the design of an information system for iterative CAs. While bidders need to decide after every round which bids to increase and by how much, given their valuations (which is private information), they typically lack information to be able to overcome the coordination and the threshold problem. Hence, our main contribution is the development of an information system that processes the bids, produces detailed information on their status and prospects, and shares this with the bidders as so-called feedback after each round. We study the effect of different types of feedback on bidder behavior and auction performance by reporting the outcome of an experiment involving over 300 individuals. By varying feedback from providing only basic information to a more substantial type of feedback (called coalitional feedback), we are able to draw (statistically significant) conclusions on how our information system impacts auction performance.

The paper is organized as follows. In the next section, we provide an overview of relevant literature dealing with either feedback and/or experimental settings in CAs. Section 3 gives the precise terminology. We describe a new type of feedback (coalitional feedback) in Sect. 4. In Sect. 5, we discuss the details of the iterative CA used in our laboratory experiments, along with the experimental design; the results are presented in Sect. 6.

\section{Related Literature}

CAs are big business, having several practical applications ranging from the allocation of airport landing slots (Rassenti et al. 1982) or harbor time slots (Ignatius et al. 2014), the allocation of spectrum licenses (Brunner et al. 2010; Scheffel et al. 2012; Bichler et al. 2013), the allocation of mineral/oil drilling rights (Cramton 2007), and real estate (Goossens et al. 2014).

An early form of feedback is described by Banks et al. (1989). They introduce a so-called "stand-by queue", which allows bidders to publicly announce their willingness to pay a certain price for a specific package. Bidders can then use this information to express a bid which, combined with one or more of the bids on the stand-by queue, is able to outbid the currently winning bid. While a stand-by queue can help to overcome the coordination problem, it is less clear how it alleviates the threshold problem. Nevertheless, there is some experimental evidence suggesting that bidders were indeed able to coordinate their bids using the stand-by queue and displace large 
package bids when the sum of the small bidders' valuations was higher than that of the large bidder (Bykowsky et al. 2000).

Adomavicius and Gupta (2005) introduce several important concepts concerning feedback: deadness and winning levels. In short, the former is the price a bidder needs to bid to have any chance of becoming winning, whereas the latter is the price that guarantees the bidder to become winning if no other bids are increased. Their work serves as a foundation for bidder support systems in CAs, as it provides theoretical, algorithmic, and computational results on deadness and winning levels.

Adomavicius et al. (2012) study how bidders behave in continuous CAs. Their main objective is to study how information feedback affects bidding behavior leading to differences in the retained surplus of bidders. They used baseline feedback (all bids displayed anonymously), outcome feedback (provisional winning allocation) and price feedback (deadness and winning levels). They find that price feedback leads to higher efficiencies, fewer dead bids and a higher percentage of winning bids when compared to outcome and baseline feedback.

Petrakis et al. (2013) build on the work by Adomavicius and Gupta (2005) and define and analyze computational and game theoretical properties of deadness and winning levels. They mention the threshold problem, and the fact that often winning levels are too high for a single bidder to outbid a large bidder. As a solution they briefly suggest coalitional winning levels, which they introduce as personalized and non-linear ask prices in between deadness and winning levels. The underlying idea is that the costs to outbid the currently winning bid is shared among the bidders in a losing coalition. However, they point out that coalitional winning levels are computationally very challenging and do not change the free-rider incentive, and as such they do not further expand on this concept. There is game-theoretical research on this free-rider problem in ascending CAs (see e.g., Sano 2012; Goeree and Lien 2014; Guler et al. 2016), that shows that ascending CAs can lead to inefficient perfect Bayesian equilibria with riskneutral bidders. However, laboratory experiments have shown that ascending combinatorial auctions consistently achieve high allocative efficiencies, indicating that this negative theoretical result does not seem to apply in practical situations.

In a time-wise overlapping but independent study, Bichler et al. (2017) introduce an ascending CA which implements coalitional winning levels, where the cost sharing is based on the Shapley value. It is important to realize an essential difference with this paper: the coalitional winning levels in Bichler et al. (2017) are implemented as a price rule. This means that bidders either accept the suggested price, or are forced to drop out. In our laboratory experiment (see Sect. 5), we use coalitional winning levels as feedback, i.e., purely informative (bidders can bid any price they prefer). Besides numerical experiments, Bichler et al. (2017) perform lab experiments with human participants on auction settings similar to the one used by Adomavicius et al. (2012), allowing them to compare results and to express causal statements about the differences. The results of their experiments indicate high economic efficiencies in ascending CAs with deadness and winning level feedback, but even higher efficiencies if the price rule based on coalitional winning levels is enforced, in addition to giving deadness and winning levels. The price rule also appears to lead to faster auctions. We position this paper as a different set of experiments, with different types of coalitional feedback in a different experimental setting. Therefore, comparing our results with these prior papers is impracticable.

\section{Notation, Terminology, and Modeling}

Our setting is an ascending, iterative CA consisting of multiple rounds. Consider a set $I=\{1,2, \ldots, m\}$ of indivisible, unique items which are auctioned using a first price (i.e., winning bidders pay the prices they bid) iterative CA, and a set $A=\{1,2, \ldots, n\}$ of bidders that participate in the CA. A bid $b$ consists of three components: the bidder $a(b) \in A$ expressing bid $b$, the package $S(b) \subseteq I$ to which bid $b$ applies, and the price $p(b)$ that bidder $a(b)$ communicates for package $S(b)$. Hence, we see a bid $b$ as a triple $(a(b), S(b), p(b))$, and we denote the set of bids by $\mathcal{B}=$ $\{(a(b), S(b), p(b)) \mid$ bidder $a(b)$ has expressed the willingness to pay $p(b)$ for package $S(b)\}$. Every bidder $a \in A$ has a value $v_{a}(S) \geq 0$ for every subset $S \subseteq I$; this value $v_{a}(S)$ is the private valuation of bidder $a$ for the package $S$.

The winner determination problem (WDP) is now the following: given the set of bids $\mathcal{B}$, determine the allocation of items to bidders that maximizes the sum of the prices of the accepted bids, ensuring that each item is sold at most once. An integer programming formulation of WDP, using binary variables $x(b)$ which are equal to one if and only if bid $b \in \mathcal{B}$ is selected as a winning bid, is presented below.

(WDP)

$$
\begin{aligned}
& \max \sum_{b \in \mathcal{B}} p(b) x(b) \\
& \text { s.t. } \sum_{b \in \mathcal{B}: i \in S(b)} x(b) \leq 1 \quad \forall i \in I \\
& x(b) \in\{0,1\} \quad \forall b \in \mathcal{B}
\end{aligned}
$$

Given a set of bids $\mathcal{B}$, an instance of the WDP follows. For each solution $x$ of this WDP, there is an allocation $X=$ $\{(a(b), S(b)) \mid x(b)=1\}$ indicating which bidder receives what set(s) of items, and a corresponding set of winning bids $\mathcal{W}(X)=\{b \in \mathcal{B} \mid x(b)=1\}$. Let $X^{*}$ and $\mathcal{W}^{*}$ symbolize 
respectively the allocation and the set of winning bids corresponding to an optimal solution of the WDP. The optimal objective function value is denoted by $W D P(I)=\sum_{b \in \mathcal{B}} p(b) x(b)^{*}$, with $x(b)^{*}$ denoting the optimal values for the decision variables.

The value of an allocation $X$ depends on the private valuations of the bidders, and is denoted by $V(X)=\sum_{b \in \mathcal{W}(X)} v_{a(b)}(S(b))$. This value can be seen as being distributed over the auctioneer on the one hand, and the bidders on the other hand. We use the term auctioneer surplus of an allocation $X$, denoted by $A S(X)$, to represent the revenue for the auctioneer: $A S(X)=\sum_{b \in \mathcal{W}(X)} p(b)$, which corresponds to the amount received by the auctioneer. The bidders surplus of an allocation $X$, denoted by $B S(X)$, is defined as $B S(X)=\sum_{b \in \mathcal{W}(X)}\left(v_{a(b)}(S(b))-p(b)\right)$. Clearly, $V(X)=A S(X)+B S(X)$.

A particular allocation, called $X^{E}$, is found when each bidder bids his/her private valuation on each possible package. The private valuations are an upper bound on the bid prices in pay-what-you-bid auctions. Hence, the value of this allocation, $V\left(X^{E}\right)$ is maximum over all allocations, and we use this quantity to be able to define the economic efficiency of any allocation $X: E(X)=V(X) / V\left(X^{E}\right)$. Notice that $0 \leq E(X) \leq 1$. The economic efficiency of an auction measures the total amount of achieved surplus relative to the maximum obtainable surplus. It represents a measure of social welfare. When the efficiency is $100 \%$, no participant in the auction, whether they are a bidder or the auctioneer, can improve their situation without making some other participant worse off. However, when the efficiency is below $100 \%$, there is still 'money left on the table'.

We refer to Adomavicius and Gupta (2005) for the original treatment of the concepts presented in this paragraph. Observe that in the course of an iterative, ascending $\mathrm{CA}$, a bid $b \in \mathcal{B}$ can be in one of three states. The state of a bid $b$ depends on its corresponding price $p(b)$. When we vary $p(b)$ from a low value, say 0 , to a high value, the state of the bid will start in a dead state, where the bid is currently not winning and has no chance of ever becoming a winning bid. Next, at a specific value for $p(b)$ called the deadness level, the state will become live; the bid is currently not winning, but it could become winning in a following round, depending upon the new bids. Finally, at another specific value called the winning level, the state will become winning (i.e., the bid is provisionally winning). This is depicted in Fig. 1.

The deadness level for a subset of items $S \subseteq I$, called $D L(S)$, is defined as the minimum price $p_{\min }$, ceteris paribus, that some bidder $a \in A$ has to bid on $S$ such that the resulting bid $\left(a, S, p_{\min }\right)$ can become winning in some future round.

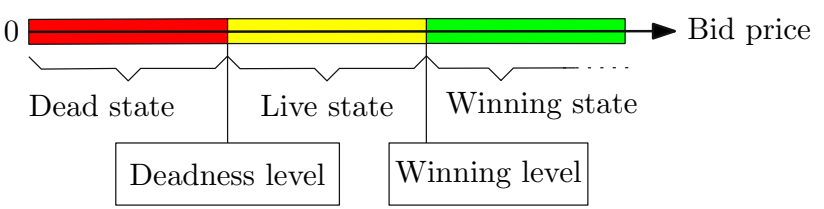

Fig. 1 Bid states

The winning level for a subset of items $S \subseteq I$, called $W L(S)$, is defined as the minimum price that some bidder $a \in A$ has to bid on package $S$ so that that bid, ceteris paribus, will become winning in the next round.

\section{Coalitional Feedback}

In this section, we discuss two types of coalitional feedback. The section closes with a numerical example.

\subsection{Factual Coalitional Feedback}

In this section, we describe a new type of feedback, called factual coalitional feedback (FCFB), which is explicitly designed to overcome coordination problems in CAs. The value of FCFB lies in the information we provide to a bidder with a non-winning bid regarding the existence of other bids that potentially can help to jointly become (provisionally) winning. This information gives a bidder an idea whether or not there is still potential in raising the bid price of the currently non-winning bid. FCFB answers the question "how many other bids exist that complement my bid, and what is, ceteris paribus, the additional amount needed?"

We now describe how to obtain factual coalitional feedback. First, consider a non-winning bid $b \in \mathcal{B}$. When calculating the winning level for $b$, i.e., $\operatorname{WL}(S(b))$, we also find a coalition of bids, referred to as $N(b)$, that consists of 'newcomers', i.e., bids that were not winning before, but become winning together with $b$. More formally, this coalition is defined as $N(b)=\left(b \cup \mathcal{W}^{*}(I \backslash S(b))\right) \backslash \mathcal{W}^{*}$, and the number of bids in that coalition is $|N(b)|$. Next, if $|N(b)|>1$, the following message goes out to all bids in $N(b)$ : "If $|N(b)|$ bids, including this one, are collectively raised by $(\mathrm{WL}(S(b))-p(b))$, these $|N(b)|$ bids become winning." Thus, all bidders in $|N(b)|$ face the same "increment', i.e., $(\mathrm{WL}(S(b))-p(b))$. A single bid receives such a feedback message each time it appears in an allocation that makes some non-winning bid winning, thus it is possible to receive multiple such messages for a single bid.

Clearly, FCFB is a potential remedy against coordination problems. Indeed, when deciding upon a new bid, a bidder can now consider, in addition to their private 
valuation, the size of the coalition, and the suggested 'increment'.

\subsection{Suggestive Coalitional Feedback}

Suggestive coalitional feedback (SCFB), goes one step further than FCFB, and adds a concrete bid suggestion in addition to the feedback given with FCFB. As such, SCFB is designed to combat both coordination and threshold problems. With SCFB, a bid $b$ for a set of items $S(b)$ will receive feedback in the following manner: "If $|N(b)|$ bids, including this one, are collectively raised by $(\mathrm{WL}(S(b))-p(b))$, these $|N(b)|$ bids become winning. We suggest you bid $p(b)+(\mathrm{WL}(S(b))-p(b)) /|N(b)| . "$ The same message also goes out to the other bidders in $N(b)$. Suggestive coalitional feedback solves the questions "are there other bids that complement my bid?" and "what price should I bid, so that I become winning instead of the currently winning bid(s)?" There are many ways to give a concrete bid suggestion and they all have advantages and disadvantages. For example, one could look at the current bid prices and suggest an amount proportional to that. The idea is then to suggest a higher bid price to bids that are high already. The disadvantage of this approach is that a bidder with a relatively low private value, that bids relatively close to this private value, can get a suggestion that is too high. Another approach is to take into account the number of items in the bids, or even incorporate the Shapley value (Bichler et al. 2017). However, these sort of technicalities often make it unnecessarily difficult for bidders to understand what is going on in the feedback. For that reason, we opted for a concrete suggestion that divides the increment equally among the bidders in the coalition. We remark that this may not always be perceived as fair, e.g., for coalitions consisting of bidders where the ratio of the highest to lowest bid price is very high. However, for the valuations we use (described in Sect. 5.2.2), this ratio is never extreme. Note that it is again possible to receive multiple feedback messages for a single bid, one message for every time it occurs in an allocation that makes some non-winning bid winning. Also note that it is possible that the concrete bid suggestion exceeds the bidder's private valuation for the corresponding set of items. In practice this is unavoidable; the auctioneer does not know the private valuations.

Finally note that coalitional feedback, both the factual and the suggestive variants, give no concrete suggestion as to which package of items to bid on. Instead, it takes into account previously made bids, and informs the bidder about coordination opportunities, and in case of suggestive coalitional feedback adds a price suggestion. In other words: bidders still need to find packages that are of interest to them (i.e., where they can get a positive bidders surplus), but coordination and cooperation with other bidders becomes easier. Receiving multiple (viable) feedback messages for one bid could be interpreted by the bidder as a realistic prospect of becoming a winner with that package, and persuade him/her to raise his/her bid on that package, rather than on other packages for which no coalitional feedback message was received. Nevertheless, the number of potential coalitions also needs some consideration. Given that the number of possible coalitions is exponential in the number of bids, the number of potential feedback messages is of the same order. Depending on the setting, limiting the number of coalitions, e.g., by considering coalitions of live bids and/or some number of 'most promising coalitions' (i.e., those with the lowest required increments), can make sense.

\subsection{Numerical Example}

Consider some round in an iterative ascending combinatorial auction with 6 bidders and 3 items (A, B, and C). The set of bids is presented in Table 1 . The columns respectively contain the bidder $a(b)$, the bid price $p(b)$, the package $S(b)$, the deadness level $D L(S(b))$, and finally the winning level $W L(S(b))$. A * in the deadness level column indicates that the bid currently is not dead (i.e., the bid is live). $\mathrm{A} *$ in the winning level column indicates that the bid is currently winning.

Bidder 1 has the (provisionally) winning bid. Bidders 2 to 5 each have live bids, so any of these could be picked up in a winning coalition. Bidder 6's bid is a dead bid and will hence never be part of any winning allocation: there is always a better alternative to selecting the bid by bidder 6 .

Bidder 2's bid will receive the following factual coalitional feedback: "If 2 bids, including this one, are collectively raised by 10 , these 2 bids become winning." It is not hard to see that the coalition induced by bidder 2's bid consists of that bid and bidder 5's bid. In fact, bidder 5 will receive the same message. There is, however, a second potentially viable coalition that consists of the bids by

Table 1 A set of bids and their corresponding deadness levels and winning levels

\begin{tabular}{lllll}
\hline$a(b)$ & $p(b)$ & $S(b)$ & $\mathrm{DL}(S(b))$ & $\mathrm{WL}(S(b))$ \\
\hline 1 & 80 & $\{\mathrm{~A}, \mathrm{~B}, \mathrm{C}\}$ & $80^{*}$ & $80^{*}$ \\
2 & 50 & $\{\mathrm{~A}, \mathrm{~B}\}$ & $50^{*}$ & 60 \\
3 & 20 & $\{\mathrm{~A}\}$ & $20^{*}$ & 40 \\
4 & 20 & $\{\mathrm{~B}\}$ & $20^{*}$ & 40 \\
5 & 20 & $\{\mathrm{C}\}$ & $20^{*}$ & 30 \\
6 & 30 & $\{\mathrm{~A}, \mathrm{~B}\}$ & 50 & 60 \\
\hline
\end{tabular}


bidders 3,4 , and 5 . They will get the following factual coalitional feedback message: "If 3 bids, including this one, are collectively raised by 20 , these 3 bids become winning." Bidders 2,3 , and 4 are each present in one viable coalition, and hence receive one feedback message. Bidder 5's bid, however, is present in two viable coalitions, and will receive two messages corresponding to those coalitions: a smaller coalition consisting of 2 bids that faces an increment of 10 and a larger coalition consisting of 3 bids that faces an increment of 20 .

\section{Methodology}

\subsection{Experimental Design}

To experimentally study the effect of feedback on the bidders' ability to overcome coordination and threshold problems, we set up iterative CAs in a lab using the z-Tree software (Fischbacher 2007). In these auctions, bidders compete to acquire a number of items, and are allowed to bid on any subset of the items (see Sect. 5.2.1). We impose no limit on the number of bids that a bidder can submit, nor do we impose any activity rules. We use a minimum bid increment of 1 , and only allow bids lower than or equal to the relevant private valuation. This eliminates gaming behavior; bidders can no longer incur possible losses. More details on the private valuations is given in Sect. 5.2.2.

We opted for an OR-bids bidding language, which means that given a number of bids from a bidder, the auctioneer can accept any non-overlapping set of these bids and charge the sum of the specified prices (see e.g., Nisan 2000). The XOR-bidding language would be a more expressive alternative, but, as stated in both Brunner et al. (2010) and Scheffel et al. (2012), XOR-bidding can lead to problems if bidders only submit few bids. Indeed, a low number of bids often leads to a number of unsold items, which may have a considerable impact on efficiency. Since we use super-additive valuations in our laboratory experiments, the OR-bids bidding language is well suited.

The auction proceeds in rounds, until two consecutive rounds occur in which the total auction revenue does not increase compared to the previous round. In other words, if three consecutive auction rounds lead to the same revenue, the auction closes. When that happens, the provisionally winning allocation becomes the final winning allocation. This closing rule effectively eliminates sniping strategies, where bidders suddenly make (higher) bids in the last round. On the other hand, it might lead to an extreme number of rounds, as bidders could still opt to do nothing as long as the auction has not resulted in consecutive rounds with the same revenue. However, in our experiments we encountered no such adverse effects.
The experimental design is presented in Table 2, and involves 3 factors: structure (STR), valuation (VAL), and feedback (FB). We refer to Sect. 5.2 for a detailed discussion of these factors. A row in Table 2 corresponds to an experimental session. An experimental session consists of 4 groups, one for every level of the factor structure. Every group in an experimental session is called an experimental unit. An experimental unit consists of a series of 4 consecutive auctions with the same set of participants, and contains 1 auction for each feedback level. Each entry in Table 2 corresponds to an auction; for instance the entry " $3 ; 4$ " refers to an auction where the factor valuation equals 3 , and the factor feedback equals 4 . A total of 192 auctions were held. Every session requires 27 subjects (one experimental unit consisting of 4 subjects, two experimental units consisting of 7 subjects, and one experimental unit consisting of 9 subjects), hence the total number of required participants for 12 sessions is 324 .

The design is between-subject for the factor structure, and within-subject for the factors valuation and feedback. In addition, all 24 permutations of the 4 feedback levels occur exactly twice, all threshold levels occur at least once per experimental unit and any two consecutive valuation levels within an experimental unit are distinct. We note that specifically in the experimental units corresponding to STR4, there are no auctions using a VAL level of 3.

A printout of the instructions (which can be found in the appendix, available online via http://www.springerlink. com) was handed out to every participant in the beginning of the experiment. All participants worked their way through the instructions and filled in a set of test questions. Participants were free to ask questions. Once all subjects were done filling in the test questions, and when all those questions were answered correctly, the auctions started. In every session, the same experimenter was present, and all the experiments were held in the same room. Students received a bonus point on the exam of a course they had to take for showing up, and a monetary incentive that depended on performance in the auctions. Performance is measured by the difference between the private values and the prices paid for the final winning bids. On average, the participants earned $€ 9.62$.

\subsection{Factors of the Experiment}

In this section we discuss the different factors (independent variables) in our experimental design.

\subsubsection{The Factor Structure (STR)}

We use four different item/bidder structures, as shown in Fig. $2 \mathrm{a}$ and $\mathrm{b}$. The item structures are similar to settings in Kazumori (2010), Scheffel et al. (2011), and Vangerven 
Table 2 Experimental sessions: first number in an experimental unit represents the VAL level, second number represents the FB level

\begin{tabular}{|c|c|c|c|c|c|c|c|c|c|c|c|c|c|c|c|c|}
\hline \multirow{2}{*}{$\frac{\text { Session }}{1}$} & \multicolumn{4}{|c|}{ STR1 } & \multicolumn{4}{|c|}{ STR2 } & \multicolumn{4}{|c|}{ STR3 } & \multicolumn{4}{|c|}{ STR4 } \\
\hline & $1 ; 1$ & $2 ; 2$ & $1 ; 3$ & $3 ; 4$ & $2 ; 3$ & $3 ; 4$ & $1 ; 1$ & $3 ; 2$ & $1 ; 4$ & $3 ; 3$ & $1 ; 2$ & $2 ; 1$ & $1 ; 3$ & $2 ; 4$ & $2 ; 2$ & $2 ; 1$ \\
\hline 2 & $1 ; 2$ & $2 ; 3$ & $1 ; 4$ & $3 ; 1$ & $2 ; 1$ & $1 ; 3$ & $3 ; 2$ & $2 ; 4$ & $1 ; 2$ & $2 ; 4$ & $3 ; 3$ & $1 ; 1$ & $2 ; 2$ & $1 ; 4$ & $2 ; 1$ & $1 ; 3$ \\
\hline 3 & $1 ; 3$ & $3 ; 4$ & $2 ; 1$ & $3 ; 2$ & $2 ; 4$ & $3 ; 2$ & $2 ; 3$ & $1 ; 1$ & $3 ; 4$ & $2 ; 3$ & $3 ; 1$ & $1 ; 2$ & $1 ; 2$ & $2 ; 1$ & $2 ; 3$ & $1 ; 4$ \\
\hline 4 & $1 ; 4$ & $3 ; 2$ & $1 ; 1$ & $2 ; 3$ & $3 ; 1$ & $2 ; 4$ & $1 ; 3$ & $2 ; 2$ & $2 ; 1$ & $1 ; 4$ & $2 ; 3$ & $3 ; 2$ & $1 ; 1$ & $2 ; 3$ & $1 ; 2$ & $2 ; 4$ \\
\hline 5 & $2 ; 1$ & $1 ; 2$ & $3 ; 3$ & $2 ; 4$ & $2 ; 2$ & $1 ; 4$ & $3 ; 1$ & $1 ; 3$ & $2 ; 2$ & $1 ; 3$ & $2 ; 1$ & $3 ; 4$ & $2 ; 1$ & $2 ; 3$ & $2 ; 4$ & $1 ; 2$ \\
\hline 6 & $2 ; 2$ & $1 ; 1$ & $2 ; 4$ & $3 ; 3$ & $3 ; 2$ & $2 ; 1$ & $3 ; 3$ & $1 ; 4$ & $1 ; 3$ & $3 ; 2$ & $1 ; 1$ & $2 ; 4$ & $1 ; 4$ & $2 ; 1$ & $1 ; 3$ & $2 ; 2$ \\
\hline 7 & $2 ; 3$ & $3 ; 1$ & $2 ; 2$ & $1 ; 4$ & $3 ; 3$ & $1 ; 2$ & $2 ; 1$ & $3 ; 4$ & $1 ; 1$ & $3 ; 4$ & $2 ; 2$ & $3 ; 3$ & $2 ; 1$ & $2 ; 2$ & $1 ; 4$ & $2 ; 3$ \\
\hline 8 & $2 ; 4$ & $3 ; 3$ & $1 ; 2$ & $2 ; 1$ & $3 ; 4$ & $1 ; 1$ & $2 ; 2$ & $3 ; 3$ & $3 ; 2$ & $2 ; 1$ & $1 ; 4$ & $2 ; 3$ & $2 ; 3$ & $2 ; 4$ & $2 ; 2$ & $1 ; 1$ \\
\hline 9 & $3 ; 1$ & $2 ; 4$ & $3 ; 2$ & $1 ; 3$ & $1 ; 2$ & $2 ; 3$ & $1 ; 4$ & $3 ; 1$ & $2 ; 3$ & $1 ; 1$ & $3 ; 2$ & $1 ; 4$ & $2 ; 4$ & $2 ; 2$ & $1 ; 1$ & $2 ; 3$ \\
\hline 10 & $3 ; 2$ & $1 ; 4$ & $2 ; 3$ & $1 ; 1$ & $1 ; 4$ & $3 ; 1$ & $1 ; 2$ & $2 ; 3$ & $3 ; 1$ & $2 ; 2$ & $3 ; 4$ & $1 ; 3$ & $2 ; 3$ & $1 ; 1$ & $2 ; 4$ & $2 ; 2$ \\
\hline 11 & $3 ; 3$ & $2 ; 1$ & $3 ; 4$ & $1 ; 2$ & $1 ; 3$ & $2 ; 2$ & $3 ; 4$ & $2 ; 1$ & $2 ; 4$ & $3 ; 1$ & $1 ; 3$ & $2 ; 2$ & $2 ; 4$ & $1 ; 2$ & $2 ; 3$ & $2 ; 1$ \\
\hline 12 & $3 ; 4$ & $1 ; 3$ & $3 ; 1$ & $2 ; 2$ & $1 ; 1$ & $3 ; 3$ & $2 ; 4$ & $1 ; 2$ & $3 ; 3$ & $1 ; 2$ & $2 ; 4$ & $3 ; 1$ & $2 ; 2$ & $1 ; 3$ & $2 ; 1$ & $2 ; 4$ \\
\hline
\end{tabular}

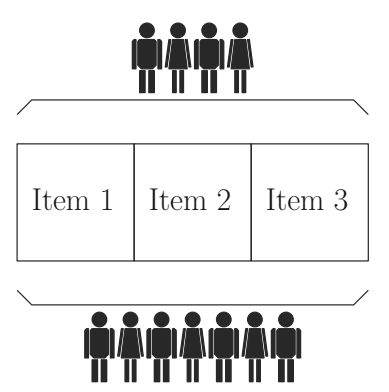

(a) 3 items and either 4 or 7 bidders.

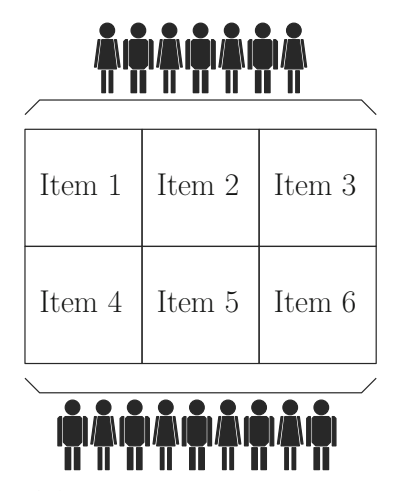

(b) 6 items and either 7 or 9 bidders.

Fig. 2 Item and bidder structure

et al. (2017). Bidders need not bid on sets of adjacent items, however, their valuations (see Sect. 5.2.2) are such that if complementarity effects exist, they involve adjacent items.

Combining both the number of items and bidders, we obtain what we refer to as the factor structure. The factor structure has four levels: 3 items with 4 bidders (STR1), 3 items with 7 bidders (STR2), 6 items with 7 bidders (STR3), and 6 items with 9 bidders (STR4).

We remark that subjects are randomly assigned to an auction, and stay in the same level of the factor structure during four consecutive auctions. In other words: four consecutive auctions in a session with the same level of structure have the same subjects.

\subsubsection{The Factor Valuation (VAL)}

With the valuation structure, we aim to obtain auction settings that give rise to coordination and threshold problems. For each structure, there are a number of small bidders who are interested in different items and 1 large bidder

who is mainly interested in a package containing all items. Each small bidder has one favorite item. The valuations of the other individual items depend on how close they are to that item: valuations for adjacent items decrease by $50 \%$ with each step they are further away from the item of main interest. The valuations for the small bidders are purely additive. The large bidder's valuation for the complete package is generated first. With super-additivities of $20 \%$ for every additional adjacent item, we calculate the valuations for all possible subsets of items. The large bidder's valuation for the complete package equals the sum over all individual items of their highest valuation by a small bidder, multiplied with a factor $W$. We discern three levels for the valuation factor, depending on $W$.

- VAL1: $W \in[106 \%, 108 \%]$. The large bidder has the upper hand, hence, no coordination is required to obtain an efficient outcome. We expect coalitions of small bidders to face a threshold problem they cannot overcome, since their valuations are not high enough. Still, it remains interesting to see how far the small bidders will boost the price for the large bidder.

- VAL2: $W \in[93 \%, 95 \%]$. Here, coalitions of smaller bidders have a small advantage over the large bidder. We expect a difficult threshold problem. Coordination is required to obtain an efficient allocation.

- VAL3: $W \in[80 \%, 82 \%]$. An efficient allocation requires small bidders to coordinate. However, as the valuation of such coalition amply exceeds the valuation of the large bidder, we anticipate an easy threshold problem.

With the resulting valuations, we avoid situations where the equal split rule used in the suggestive coalitional feedback creates situations that can be perceived as unfair. We created two sets of private values for every 
combination of STR and VAL, leading to a total of 24 different sets of private values. ${ }^{1}$

Finally, we remark that participants are not told their role (e.g., small bidder) or that of others; they simply discover their private valuations by clicking on packages. The values are private, but participants are informed that all values are at least additive. Participants take part in consecutive auctions, but valuations (roles) rotate between these auctions.

\subsubsection{The Factor Feedback (FB)}

The third factor in our laboratory experiments is feedback. Feedback is calculated after every round and communicated to the bidders. We use a hierarchy of feedback involving four levels, as depicted in Fig. 3. The first level, outcome feedback (FB1), consists of showing the (provisionally) winning allocation along with the prices corresponding to that allocation. The second feedback level (FB2) consists of the feedback given in FB1, and adds winning and deadness levels. We call this bid states feedback. Another layer up the hierarchy, in FB3, we add factual coalitional feedback on top of the feedback given in FB2. The fourth feedback level (FB4) adds a concrete bid suggestion, suggestive coalitional feedback, in addition to the feedback given in FB2. Feedback regarding a particular subset of the items is displayed if the bidder clicks on that subset.

Our coalitional feedback bears some resemblance to the price rule based on coalitional winning levels used in Bichler et al. (2017). As discussed in Sect. 2, there are a number of differences on the implementation level. (1) In Bichler et al. (2017), the coalitional pricing rule is calculated for currently losing bidders, but in their experiments the price rule for a bid follows from one coalition, specifically the coalition with the lowest price suggestion for that bid. In our experiments, we allow for multiple messages to be displayed, because it is possible that the lowest suggested amount corresponds to a coalition that faces a threshold they simply cannot bridge given their valuation. Moreover, we want to make sure that when one coalition member receives a price suggestion, all other members whose collaboration is required also receive this suggestion. (2) In our versions of coalitional feedback we only consider coalitions of live bids for feedback. Disregarding coalitions that include dead bids has the advantage of limiting the number of feedback suggestions, and requiring bidders to first bid past their respective deadness

\footnotetext{
$\overline{1}$ The private valuations used in the experiment are available here: https://feb.kuleuven.be/public/u0093797/Valuations/. Accessed 25 Feb 2020.
}

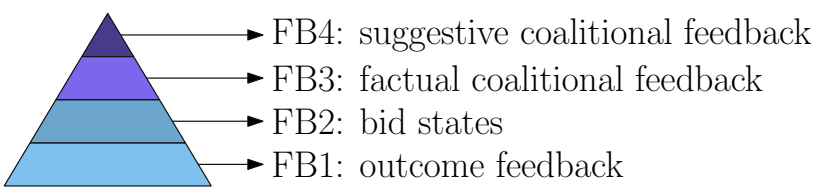

Fig. 3 Feedback hierarchy

levels. It also encourages activity in the auction, without actually requiring an explicit activity rule.

\section{Results}

We report the results of 192 auctions, involving 324 different subjects (students at the Faculty of Economics and Business), carried out at KU Leuven.

\subsection{Validation}

Before we start discussing the outcome of the experiment, it makes sense to first validate whether the experimental design resulted in the coordination and threshold problems we anticipated, and whether it aligns with what is reported in the literature on cognitive limits.

\subsubsection{The Realized Coordination and Threshold Problem}

In order to validate whether the experimental settings indeed led to coordination and threshold problems, we make use of measures for both problems described in Vangerven (2017). The Coordination Index (CI) is a measure for the magnitude of the coordination problem, and basically looks at the total relative loss in economic efficiency if each bidder bids his/her true valuation on his/ her $k$ most valuable packages, for $k$ ranging from 1 to a value $k_{\max }$ for which an efficient allocation is obtained. The larger CI, the larger the coordination challenge present in the auction. The Threshold Index (TI) for a coalition of non-winning bids boils down to the ratio of the price increase that this coalition jointly needs in order to win, to the margin this coalition has, taking into account the private valuations and current bid prices. A higher value for TI corresponds to a more severe threshold problem. If TI $>1$, the non-winning coalition cannot outbid the currently winning bids (without exceeding private valuations); this situation is called an insurmountable threshold problem.

We have computed the realized CI and TI values for each auction. Since we found that auctions using STR1 and STR2 (3 items) are very similar with respect to CI and TI, we have grouped them. The same applies for STR3 and STR4 (6 items). Moreover, for auctions with VAL1, all 
Table 3 Mean CI; TI values for each combination of the factors STR and VAL

\begin{tabular}{lllllll}
\hline & VAL1 & & VAL2 & \multicolumn{3}{c}{ VAL3 } \\
\hline STR1-2 & 0.00 & 0.98 & 0.20 & 0.25 & 0.63 & 0.12 \\
STR3-4 & & & 1.96 & 0.24 & 7.73 & 0.09 \\
\hline
\end{tabular}

item/bidder structures resulted in similar TI and CI values. The average CI and TI values are given in Table 3.

Since the highest valuation in VAL1 is that of the large bidder for the complete package, it is logical that $\mathrm{CI}$ equals 0 . For STR1 and STR2, we find easy coordination challenges for both VAL2 and VAL3. On the other hand STR3 and STR4 lead to difficult coordination problems, in particular for VAL3.

Note that we calculated TI from the perspective of the small bidders, namely, we look at the highest value coalition of small bidders versus the large package bidder. The results reported in Table 3 are averaged over every auction that has such a TI value (not all auctions have TI values to compute if the coalitions mentioned above were not present). Overall, the TI values show that the threshold problems we wanted to create, were indeed present. For VAL1, we see a TI value that, on average, is almost equal to 1 . Considering that there were auctions in which the coalition of smaller bidders all bid up to their private valuation but did not win, and hence did not have a TI value, this TI value indeed seems to correspond to insurmountable threshold problems for a coalition of small bidders. For VAL2, we find an average TI value of 0.25 , and for VAL3 we find a value of 0.11 , corresponding to difficult and easy threshold problems respectively. This also looks to be in line with the expectations sketched in Sect. 5.2.2.

For the remainder of this paper, we use the following notation:

- $\mathrm{COT} \uparrow$ includes all settings with VAL1, corresponding to an insurmountable threshold problem,

- $\mathrm{C}-\mathrm{T}+$ includes all settings with VAL2 and STR1-2, corresponding to an easy coordination problem and a difficult threshold problem,

- $\mathrm{C}+\mathrm{T}+$ includes all settings with VAL2 and STR3-4, corresponding to a difficult coordination problem and a difficult threshold problem,

- $\mathrm{C}-\mathrm{T}$ - includes all settings with VAL3 and STR1-2, corresponding to an easy coordination problem and an easy threshold problem,

- $\mathrm{C}+\mathrm{T}-$ includes all settings with VAL3 and STR3-4, corresponding to a difficult coordination problem and an easy threshold problem.

\subsubsection{Cognitive Limits}

Figure 4 depicts the average number of different packages a bidder bids on per round. This excludes the bids entered in the first round, as bidders are then still discovering their private valuations and enter a lot of bids. This result confirms the findings of Kagel et al. (2010) and Scheffel et al. (2012), who observe that bidders usually bid on a limited number of different packages, independent of the auction format. Furthermore, it shows that bidder support in the form of feedback FB2-4 reduces the number of packages bidders bid on in an auction for those settings with difficult coordination problems, or if the threshold problem is insurmountable. Bidders can focus on fewer packages, and still achieve higher efficiencies and revenues compared to basic outcome feedback (see Sect. 6.2). If the coordination problem is easy, feedback does not seem to impact the number of packages that bidders track.

\subsection{Observations}

We discuss the results of our experiments in terms of economic efficiency, bid prices, auction revenue, bidders surplus, and auction duration.

\subsubsection{Economic Efficiency}

Overall, efficiencies were quite high. Figure 5 has box plots showing the efficiency per level of feedback. We see that in the cases where only basic feedback is given (FB1) efficiencies show the highest degree of dispersion. This indicates that simply showing the (provisionally) winning allocation as feedback is often insufficient for bidders to find an efficient outcome. The difference between FB1 on the one hand, and FB2-4 on the other hand is striking: it seems deadness and winning levels are important in guiding bidders to an efficient outcome. The FB2, FB3 and FB4

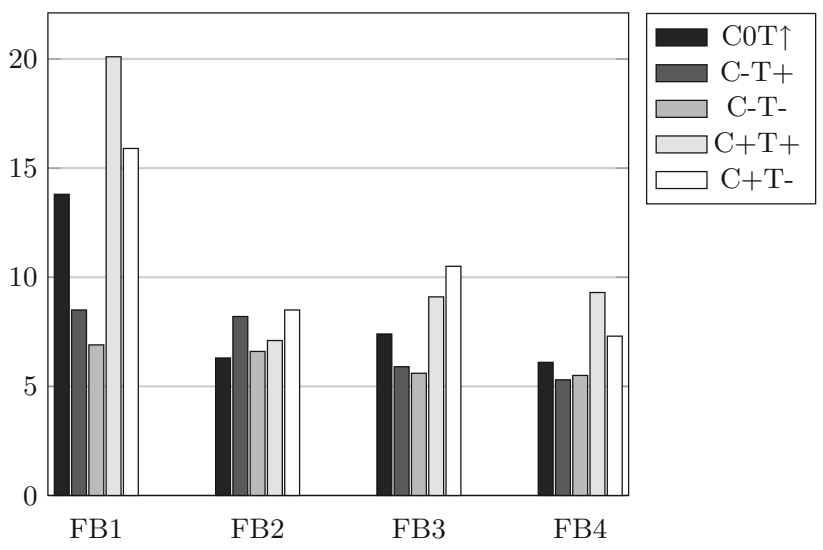

Fig. 4 Average number of bids entered per bidder per round (excluding round 1) on unique packages 


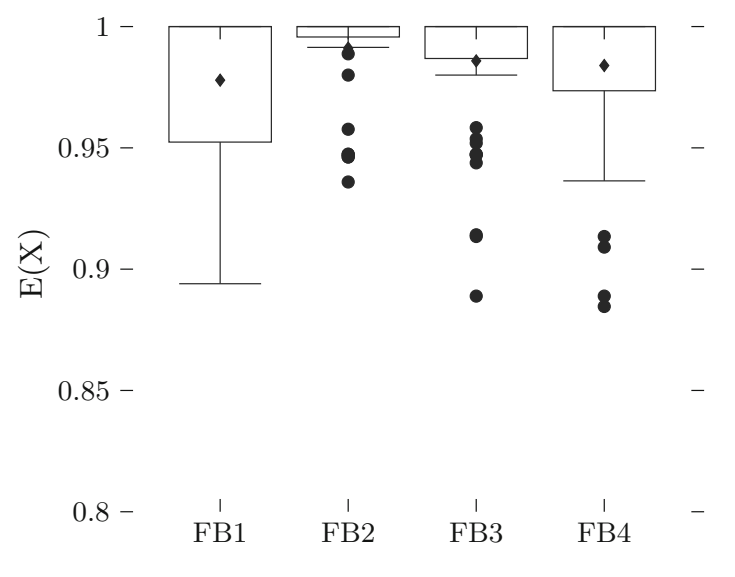

Fig. 5 FB box plots

box plots look similar, although FB3 and FB4 show a couple of outliers.

We use the non-parametric Wilcoxon-Mann-Whitney test to examine the differences in efficiencies. The notation $\prec, \prec^{*}$ and $\prec^{* *}$ respectively denote a difference at $10 \%, 5 \%$ and $1 \%$ significance level, and $\approx$ denotes we cannot reject the null hypothesis.

Onservation 1 Efficiencies ranked by Wilcoxon-MannWhitney tests:

$\mathrm{FB} 1 \prec^{* *}(\mathrm{FB} 2 \approx \mathrm{FB} 3 \approx \mathrm{FB} 4)$

At .01 significance level, we conclude that the efficiencies obtained under FBI are lower than those obtained under FB2, FB3, and FB4. At .1 significance level, we cannot reject the hypothesis that the efficiencies obtained under $F B 2, F B 3$, and FB4 come from the same distribution.

In order to obtain a more detailed understanding of these results, we check whether the degree of coordination and threshold problems has an impact. Table 4 contains the mean efficiencies per feedback level, for each of the 5 auction settings. Box plots for efficiency per feedback level for the 5 auction settings are given in the online appendix.

In the insurmountable threshold case, COT $\uparrow$, we see that efficiencies are almost always $100 \%$, i.e., the large bidders win when they should win, no matter the feedback. In the difficult threshold cases, FB3 and FB4 fare better than FB1

Table 4 Mean E(X)

\begin{tabular}{llllll}
\hline & $\mathrm{COT} \uparrow$ & $\mathrm{C}-\mathrm{T}+$ & $\mathrm{C}-\mathrm{T}-$ & $\mathrm{C}+\mathrm{T}+$ & $\mathrm{C}+\mathrm{T}-$ \\
\hline FB1 & 0.986 & 0.979 & 0.989 & 0.976 & 0.927 \\
FB2 & 0.999 & 0.985 & 1.00 & 0.978 & 0.995 \\
FB3 & 0.986 & 0.993 & 0.989 & 0.986 & 0.963 \\
FB4 & 0.988 & 0.993 & 0.975 & 0.979 & 0.982 \\
\hline
\end{tabular}

and FB2: the average efficiency is higher, and the spread is lower. This difference is striking in the case when the coordination problem is easy $(\mathrm{C}-\mathrm{T}+)$. This indicates that coalitional feedback offers added value to overcome the threshold problem. When the coordination problem is hard, FB3-4 loses its advantage compared to FB2. In the $\mathrm{C}-\mathrm{T}-$ case, FB4 actually performs worse than FB2, for which remarkably - all auctions ended efficiently. These observations are confirmed by Wilcoxon-Mann-Whitney tests.

Onservation 2 Table 5 contains the efficiencies ranked by Wilcoxon-Mann-Whitney tests.

For COT $\uparrow$, we cannot reject the hypothesis that the efficiencies obtained under FB1, FB2, FB3, and FB4 come from the same distribution.

For $C-T+$, at .1 significance level, we conclude that the efficiencies obtained under coalitional feedback (FB3 and FB4) are larger than those obtained under FB1 and FB2.

For $C-T-$, at .1 significance level, we conclude that the efficiencies obtained under FB2 are larger than those obtained under FB4.

In settings with a high coordition problem $(C+T+$ and $C+T-)$, at .1 significance level, we conclude that the efficiencies obtained under FBI are lower than those obtained under FB2, FB3 and FB4.

Figure 6 depicts the proportion of auctions that ended efficiently. Overall, FB1 shows the lowest percentage of efficient auctions, however, the difference is particularly notable for the settings with hard coordination problems (not a single auction ended efficiently in the $\mathrm{C}+\mathrm{T}-$ case). Comparing coalitional feedback (FB3 and FB4) with bid states feedback (FB2), the former performs better in the $\mathrm{C}-\mathrm{T}+$ case, and nearly identical in the cases with hard coordination problems.

\subsubsection{Bid Prices}

Table 6 presents the average ratio of the bid prices to their private valuations for all expressed bids. Table 7 contains the Wilcoxon-Mann-Whitney tests.

Table 5 Ranked efficiencies

\begin{tabular}{ll}
\hline & Wilcoxon-Mann-Whitney tests \\
\hline $\mathrm{COT} \uparrow$ & $\mathrm{FB} 1 \approx \mathrm{FB} 2 \approx \mathrm{FB} 3 \approx \mathrm{FB} 4$ \\
$\mathrm{C}-\mathrm{T}+$ & $(\mathrm{FB} 1 \approx \mathrm{FB} 2) \prec(\mathrm{FB} 3 \approx \mathrm{FB} 4)$ \\
$\mathrm{C}-\mathrm{T}-$ & $\mathrm{FB} 4 \prec \mathrm{FB} 2$ \\
$\mathrm{C}+\mathrm{T}+$ & $\mathrm{FB} 1 \prec(\mathrm{FB} 2 \approx \mathrm{FB} 3 \approx \mathrm{FB} 4)$ \\
$\mathrm{C}+\mathrm{T}-$ & $\mathrm{FB} 1 \prec(\mathrm{FB} 2 \approx \mathrm{FB} 3 \approx \mathrm{FB} 4)$ \\
\hline
\end{tabular}




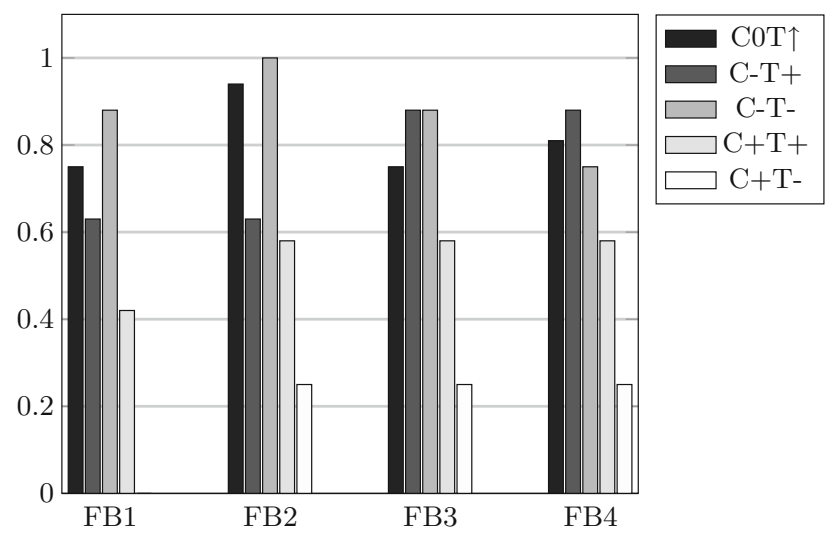

Fig. 6 Proportion of efficient auctions

Onservation 3 In COT $\uparrow$ we see that with more feedback, bidders on average bid a higher percentage of their private valuations. In cases with a difficult threshold problem $(C-T+$ and $C+T+)$, we see that when FB3-4 is given, bidders on average bid higher compared to FB1-2 cases. In this case, the coalitional feedback appears to convince bidders to bid higher. This is not the case for the settings with easy threshold problems, in fact, for $C+T$-average bid prices are lower for FB3-4, compared to when FBI-2 is given.

\subsubsection{Auction Revenue}

Table 8 contains the mean auction revenue (i.e., the auctioneer surplus). We see that revenues in auctions with an easy threshold problem $(\mathrm{C}-\mathrm{T}-$ and $\mathrm{C}+\mathrm{T}-)$ are clearly lower than in auctions with a difficult threshold problem.

Table 6 Average bid as percentage of private valuations

\begin{tabular}{llllll}
\hline & $\mathrm{COT} \uparrow$ & $\mathrm{C}-\mathrm{T}+$ & $\mathrm{C}-\mathrm{T}-$ & $\mathrm{C}+\mathrm{T}+$ & $\mathrm{C}+\mathrm{T}-$ \\
\hline FB1 & 0.812 & 0.812 & 0.821 & 0.847 & 0.840 \\
FB2 & 0.842 & 0.786 & 0.790 & 0.853 & 0.862 \\
FB3 & 0.839 & 0.811 & 0.804 & 0.863 & 0.828 \\
FB4 & 0.821 & 0.845 & 0.783 & 0.857 & 0.821 \\
\hline
\end{tabular}

Table 7 Ranked ratio of the average bid prices to private valuations

\begin{tabular}{ll}
\hline & Wilcoxon-Mann-Whitney tests \\
\hline $\mathrm{COT} \uparrow$ & $\mathrm{FB} 1 \approx \mathrm{FB} 2 \approx \mathrm{FB} 3 \approx \mathrm{FB} 4$ \\
$\mathrm{C}-\mathrm{T}+$ & $(\mathrm{FB} 1 \approx \mathrm{FB} 2 \approx \mathrm{FB} 3) \prec{ }^{*} \mathrm{FB} 4$ \\
$\mathrm{C}-\mathrm{T}-$ & $\mathrm{FB} 1 \approx \mathrm{FB} 2 \approx \mathrm{FB} 3 \approx \mathrm{FB} 4$ \\
$\mathrm{C}+\mathrm{T}+$ & $\mathrm{FB} 2 \prec \mathrm{FB} 3, \mathrm{FB} 4 \prec \mathrm{FB} 3$ \\
$\mathrm{C}+\mathrm{T}-$ & $\mathrm{FB} 3 \prec \mathrm{FB} 2$ \\
\hline
\end{tabular}

We have seen in Sect. 6.2.2 that especially in the C-Tsetting small bidders do not bid high compared to their private valuations. Indeed, in this setting they have plenty of margin to outbid the package bidder, and given the easy coordination and threshold problem, they seem to realize this quickly in the auction, which impacts the auction revenue (Table 9).

Examining the box plots of auction revenue for the various feedback levels (Fig. 7), we notice little difference between feedback levels 2, 3 and 4. Restricting the bidders to outcome feedback (FB1), however, does reduce the auction revenue, as is confirmed by Wilcoxon-MannWhitney tests.

Onservation 4 Auction revenues ranked by WilcoxonMann-Whitney tests:

$\mathrm{FB} 1 \prec^{*}(\mathrm{FB} 2 \approx \mathrm{FB} 3 \approx \mathrm{FB} 4)$

At .05 significance level, we conclude that the auction revenues obtained under FBI are lower than those obtained under FB2, FB3, and FB4. At .1 significance level, we cannot reject the hypothesis that the revenues obtained under FB2, FB3, and FB4 come from the same distribution.

In line with Observation 3, in cases with a difficult threshold problem $(C-T+$ and $C+T+)$, we see that when FB3-4 is given, revenues are higher compared to FB1-2 cases. This is not so much the case for the settings with easy threshold problems.

Table 8 Mean auction revenue

\begin{tabular}{llllll}
\hline & COT $\uparrow$ & C-T+ & C-T - & C+T + & C+T- \\
\hline FB1 & 0.929 & 0.915 & 0.850 & 0.919 & 0.844 \\
FB2 & 0.943 & 0.962 & 0.864 & 0.930 & 0.918 \\
FB3 & 0.919 & 0.951 & 0.874 & 0.937 & 0.838 \\
FB4 & 0.929 & 0.955 & 0.849 & 0.938 & 0.873 \\
\hline
\end{tabular}

Table 9 Ranked revenues

\begin{tabular}{ll}
\hline & Wilcoxon-Mann-Whitney tests \\
\hline $\mathrm{COT} \uparrow$ & $\mathrm{FB} 1 \approx \mathrm{FB} 2 \approx \mathrm{FB} 3 \approx \mathrm{FB} 4$ \\
$\mathrm{C}-\mathrm{T}+$ & $\mathrm{FB} 1 \prec^{* *}(\mathrm{FB} 2 \approx \mathrm{FB} 3 \approx \mathrm{FB} 4), \mathrm{FB} 3 \prec \mathrm{FB} 4$ \\
$\mathrm{C}-\mathrm{T}-$ & $\mathrm{FB} 1 \approx \mathrm{FB} 2 \approx \mathrm{FB} 3 \approx \mathrm{FB} 4$ \\
$\mathrm{C}+\mathrm{T}+$ & $\mathrm{FB} 1 \prec^{*} \mathrm{FB} 4$ \\
$\mathrm{C}+\mathrm{T}-$ & $(\mathrm{FB} 1 \approx \mathrm{FB} 2) \prec \mathrm{FB} 3$ \\
\hline
\end{tabular}




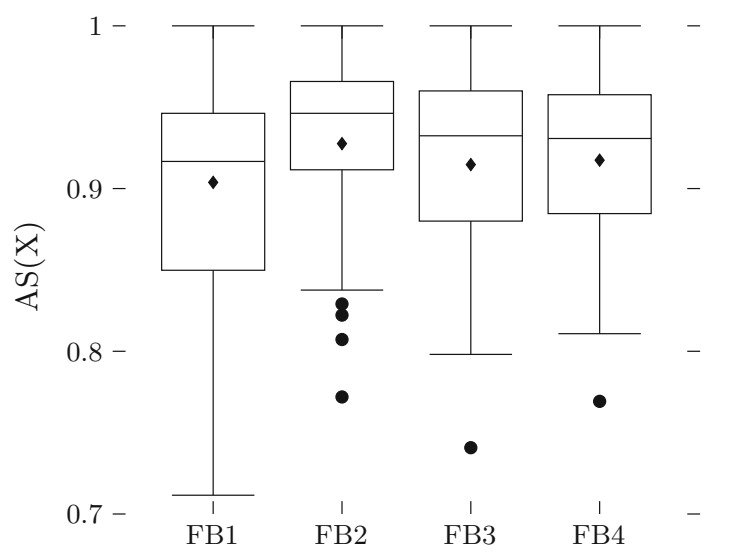

Fig. 7 Auction revenue box plots

\subsubsection{Bidders Surplus}

Table 10 contains the percentage of available surplus that goes to the bidders, i.e., $B S(X) / V\left(X^{E}\right)$ and Table 11 contains the results of the Willcoxon-Mann-Whitney tests.

Onservation 5 In the cases with difficult threshold problems, the highest bidder profits are obtained when FBI is provided. However, in these settings efficiency was much lower than with feedback FB3-4. Hence, what happened there is that large bidders received the total $B S$ when it was not economically efficient for them to receive any surplus at all. In $C-T+$, bidders obtained a larger share of the potential surplus with coalitional feedback (FB3-4),

Table 10 Average percentage of surplus obtained by the bidders $\left(B S(X) / V\left(X^{E}\right)\right)$

\begin{tabular}{llllll}
\hline & COT $\uparrow$ & C-T + & C-T - & C+T+ & C+T- \\
\hline FB1 & 0.057 & 0.064 & 0.139 & 0.057 & 0.084 \\
FB2 & 0.056 & 0.023 & 0.136 & 0.048 & 0.076 \\
FB3 & 0.067 & 0.042 & 0.115 & 0.049 & 0.125 \\
FB4 & 0.059 & 0.038 & 0.125 & 0.042 & 0.109 \\
\hline
\end{tabular}

Table 11 Ranked bidder surplus

\begin{tabular}{ll}
\hline & Wilcoxon-Mann-Whitney tests \\
\hline $\mathrm{COT} \uparrow$ & $\mathrm{FB} 1 \approx \mathrm{FB} 2 \approx \mathrm{FB} 3 \approx \mathrm{FB} 4$ \\
$\mathrm{C}-\mathrm{T}+$ & $\mathrm{FB} 2<^{*}(\mathrm{FB} 1 \approx \mathrm{FB} 3 \approx \mathrm{FB} 4)$ \\
$\mathrm{C}-\mathrm{T}-$ & $\mathrm{FB} 1 \approx \mathrm{FB} 2 \approx \mathrm{FB} 3 \approx \mathrm{FB} 4$ \\
$\mathrm{C}+\mathrm{T}+$ & $\mathrm{FB} 1 \approx \mathrm{FB} 2 \approx \mathrm{FB} 3 \approx \mathrm{FB} 4$ \\
$\mathrm{C}+\mathrm{T}-$ & $(\mathrm{FB} 1 \approx \mathrm{FB} 2) \prec \mathrm{FB} 3$ \\
\hline
\end{tabular}

compared to bid states feedback (FB2), combined with a higher efficiency (see Table 4).

\subsubsection{Auction Duration}

Table 12 contains the mean number of auction rounds for each feedback level. Auctions with FB2-4 seem to last a few more rounds on average than auctions with FB1, where not a lot of coordination is possible between bidders. Furthermore, we notice that in case of an insurmountable threshold problem (COT $\uparrow$ ), the number of rounds under FB1 is lower compared to the other settings, for all levels of feedback. Apparently, it becomes clear to the small bidders that they will not be able to win relatively quickly, compared to the other auction settings where the small bidders indeed have better odds. Furthermore, ceteris paribus, increasing the difficulty of the coordination problem increases the required number of rounds for FB3 and FB4.

The average round duration (in seconds) is provided in Table 13. In cases with a difficult coordination problem $(\mathrm{C}+\mathrm{T}-$ and $\mathrm{C}+\mathrm{T}+)$, bidders seem to need more time per round than in cases with an easy coordination problem, especially for FB1. This can be explained by the fact that bidders need to click on several itemsets to explore their options and to compose individually profitable and collectively complementary packages. In particular in the case with FB1, they receive little or no guidance for this task. Apparently, finding a promising itemset to bid on is more time-consuming than deciding on the amount with which to

Table 12 Mean number of rounds per auction

\begin{tabular}{llrccc}
\hline & $\mathrm{COT} \uparrow$ & $\mathrm{C}-\mathrm{T}+$ & $\mathrm{C}-\mathrm{T}-$ & $\mathrm{C}+\mathrm{T}+$ & $\mathrm{C}+\mathrm{T}-$ \\
\hline FB1 & 6.4 & 8.0 & 6.8 & 6.8 & 7.8 \\
FB2 & 8.5 & 10.3 & 7.8 & 8.8 & 10.5 \\
FB3 & 6.7 & 9.1 & 8.4 & 10.3 & 10.3 \\
FB4 & 6.8 & 9.1 & 11.0 & 10.2 & 17 \\
\hline
\end{tabular}

Table 13 Mean auction duration (seconds) per round

\begin{tabular}{llllll}
\hline & COT $\uparrow$ & C-T + & C-T - & C+T+ & C+T- \\
\hline FB1 & 154 & 128 & 138 & 194 & 154 \\
FB2 & 138 & 116 & 128 & 174 & 160 \\
FB3 & 184 & 113 & 125 & 151 & 145 \\
FB4 & 170 & 128 & 97 & 162 & 121 \\
\hline
\end{tabular}


increase the current bid. Apart from the setting with an insurmountable threshold problem (COT $\uparrow$ ), FB3 and FB4 manage to reduce the mean round duration.

\subsection{Discussion}

In our results, we find little difference between factual coalitional feedback (FB3) and suggestive coalitional feedback (FB4). Apparently, the price suggestion, which consists of evenly splitting the required increment among the bidders in the coalition, did not make a difference compared to simply stating the increment and the number of bidders in the coalition. One explanation could be that the participants simply came up with the same idea about the price they should bid in the next round when confronted with the factual feedback.

It should not be surprising that FB4 has added value compared to FB2 for threshold problems in cases where winning levels (WL) exceed private valuations (PV). However, if the coalitional feedback price suggestion (CFB) offered by FB4 is such that CFB is lower than WL and at the same time $\mathrm{WL}<\mathrm{PV}$, it persuades bidders towards smaller bid increments. This could either lead to an increase in the number of rounds, or - in case of an abrupt stopping rule - the auction could close with a less efficient outcome (when compared to FB2). In what follows, we provide a detailed explanation for this, for every level of Coordination/Threshold.

When the threshold problem is insurmountable (COT $\uparrow)$, aside from some outliers, feedback does not have a real impact on who will be the winning bidder. However, when FB2-4 is applied the package bidder has a harder time because the competition with the smaller bidders, even though they cannot win, is stronger. This leads to the package bidder having to bid higher prices, as is reflected in the higher auctioneer revenue results. In this case, FB3 and FB4 have no added value compared to FB2 in terms of efficiency or revenue. The reason for this is twofold: a substantial amount of the feedback messages of FB4 is either (1) "useless" (i.e., CFB > PV), as seen in Table 14 and/or (2) sent to bidders that at that time have at least one provisionally winning bid, as seen in Table 15 . In the former case bidders will not be able to follow the feedback and in the latter case bidders might not be willing to follow

Table 14 Average percentage of useful and useless FB4 messages

The remainder of the messages fall in a category where both CFB and WL are below the PV

\begin{tabular}{lll}
\hline Setting & Useless & Useful \\
\hline $\mathrm{COT} \uparrow$ & 60.3 & 23.2 \\
$\mathrm{C}-\mathrm{T}+$ & 21.4 & 33.1 \\
$\mathrm{C}-\mathrm{T}-$ & 36.7 & 13.3 \\
$\mathrm{C}+\mathrm{T}+$ & 45.7 & 25.0 \\
$\mathrm{C}+\mathrm{T}-$ & 44.9 & 9.9 \\
\hline
\end{tabular}

Table 15 Average percentage of useful FB4 messages that go out to current (non-)winners

\begin{tabular}{lll}
\hline Setting & To winners & To non-winners \\
\hline $\mathrm{COT} \uparrow$ & 56.8 & 43.2 \\
$\mathrm{C}-\mathrm{T}+$ & 54.2 & 45.8 \\
$\mathrm{C}-\mathrm{T}-$ & 81.3 & 18.8 \\
$\mathrm{C}+\mathrm{T}+$ & 43.1 & 56.9 \\
$\mathrm{C}+\mathrm{T}-$ & 75.9 & 24.1 \\
\hline
\end{tabular}

the suggestion. After all, they already have a provisional winning bid.

In cases with a considerable threshold problem, the coalitional feedback appears to convince bidders to bid higher, which leads to higher efficiencies. If FB1 is applied, the highest bidder profits are obtained: this is an indication of the threshold problem: in cases where the efficiency is not $100 \%$, the large package bidders receive the total bidders surplus while it is not economically efficient for them to receive any surplus at all. When more feedback is given, total bidder profits decrease, yet efficiencies improve as bidders are better able to coordinate and compete with the large package bidder. Indeed, one would expect FB3 and FB4 to be more effective than FB2 in cases where the winning levels are restrictively high, i.e., greater than the private values of losing bidders, and hence the information is not of much use to bidders. In such a case, FB3 and more so FB4 have the largest potential effect because of the new information they provide. This happens more often in difficult threshold cases. Indeed, in Tables 14 and 15 we respectively see that in $\mathrm{C}-\mathrm{T}+$ and $\mathrm{C}+\mathrm{T}+$ the highest proportion of messages are useful and go out to current non-winning bidders.

In easy threshold cases, FB2 performs better than FB4 with regards to efficiency. It seems that FB4 (sometimes) prevents the small bidders from bidding what they should bid to win the auction. The explanation for this phenomenon is threefold. First, FB4 is rarely an added value compared to WL. Table 14 shows that in only around 10 to $13 \%$ of the messages sent out in auctions with an easy threshold problem, the coalitional feedback has an added value compared to winning levels. Second, in the case useful coalitional feedback is sent, it most often goes out to current winners instead of non-winners, as can be seen in Table 15. Third, if useful FB4 feedback is sent out, the bidders in fact get the message that they could also win by bidding (much?) less than the winning level. If only some of the bidders in a losing coalition follow this advice, the auction requires more rounds. Moreover, given our potentially abrupt stopping rule, the auction could stop prematurely. 
Overall, with respect to speed of convergence, our coalitional feedback often provokes more rounds than similar settings with bid state or outcome feedback. In some cases, this brings an increase in efficiency or revenue. However, we notice a substantial drop in the mean duration of a round, which reflects that our feedback reduces the time the bidders spend exploring their options or contemplating their bid increment.

\section{Conclusions}

In situations where bidders have different additive or super-additive private valuations, academic literature has shown that CAs have the edge over (sequential) single item auctions. However, CAs introduce two problems: the coordination problem and the threshold problem. The coordination problem arises when bidders fail to identify bids that are individually profitable and collectively complementary. The threshold problem represents the next problem: even when individually profitable and collectively complementary packages are identified, and the coordination problem is essentially overcome, the problem of determining bid prices still remains. This is further complicated by free-rider incentives. Naturally, these problems are of significant practical interest.

We design types of feedback dubbed coalitional feedback to help bidders overcome coordination and threshold problems. We put different types of feedback to the test in a laboratory setting with human bidders, using iterative CAs. There are many ways coalitional feedback can be implemented: the number of messages could be limited, bid suggestions may be based on different ways of splitting the required increment over the coalition members, the feedback might be binding and function as a bid price rule, etc. While conceptually similar, these differences in implementation might result in different effects, depending on the auction setting (e.g., small vs. large auctions), which makes them an interesting topic for future research.

In line with Adomavicius et al. (2012), we find that bid states feedback is a big improvement upon outcome feedback, both with respect to economic efficiency and auction revenue. We find that coalitional feedback offers further improvements, leading to higher efficiencies as well as higher bidder profits, when threshold problems are difficult. The advantages include reducing the complexity bidders face with package bidding and enabling bidders to focus more on relevant packages. Furthermore, they can adjust their bid prices smartly, considering both the coalitional winning levels and the number of coalitions. We conclude that confronted with a difficult threshold problem, bidders are not insensitive to bid price suggestions and tend to follow such suggestions readily. This is interesting, as it appears that the free-rider aspect is at least diminished by coalitional feedback.

We learned that two factors are important for coalitional feedback to have an added value compared to bid states feedback. First, coalitional feedback works well if it suggests a price increment which is lower than the private valuation, while the winning level is higher than the private valuation. On the other hand, when the latter is below the private valuation, coalition feedback may have an adverse effect. Second, coalitional feedback has more potential impact when it goes out to bidders, who at the time of receiving the feedback do not have a provisionally winning bid. We have witnessed this particularly in cases with a high threshold problem.

This paper shows what can potentially be achieved using coalitional feedback. Whether or not such feedback can be implemented in practice may however depend on other considerations. Indeed, feedback is information, which will allow bidders to learn about the preferences and behaviour of other (rivaling) bidders. Despite being helpful in terms of economic efficiency or bidder profits, this could be perceived as inappropriate in some practical settings.

While our research has mainly been done with human bidders in mind, the (factual) coalitional feedback can also be offered to automated bidding agents. An automated bidding agent bids on behalf of a human bidder, according to some bidding strategy and after having learned the preferences of the human bidder. Clearly, coalitional feedback offers automated bidding agents objective information on the coalitions in which it features and the threshold that needs to be bridged by each of these coalitions. How automated bidding agents could best use this information to determine which packages are the most interesting to pursue, and how to improve current automated bidding strategies to take this into account are interesting questions for further research.

Acknowledgements Open Access funding provided by Projekt DEAL.

Open Access This article is licensed under a Creative Commons Attribution 4.0 International License, which permits use, sharing, adaptation, distribution and reproduction in any medium or format, as long as you give appropriate credit to the original author(s) and the source, provide a link to the Creative Commons licence, and indicate if changes were made. The images or other third party material in this article are included in the article's Creative Commons licence, unless indicated otherwise in a credit line to the material. If material is not included in the article's Creative Commons licence and your intended use is not permitted by statutory regulation or exceeds the permitted use, you will need to obtain permission directly from the copyright holder. To view a copy of this licence, visit http://creativecommons. org/licenses/by/4.0/. 


\section{References}

Adomavicius G, Gupta A (2005) Toward comprehensive real-time bidder support in iterative combinatorial auctions. Inf Syst Res 16(2):169-185

Adomavicius G, Curley SP, Gupta A, Sanyal P (2012) Effect of information feedback on bidder behavior in continuous combinatorial auctions. Manag Sci 58(4):811-830

Ausubel LM, Milgrom P (2006) The lovely but lonely vickrey auction. In: Cramton P, Shoham Y, Steinberg R (eds) Combinatorial auctions, chap 1. MIT Press, Cambridge, pp 17-40

Banks JS, Ledyard JO, Porter DP (1989) Allocating uncertain and unresponsive resources: an experimental approach. RAND J Econ 20(1): 1-25

Bichler M, Shabalin P, Wolf J (2013) Do core-selecting combinatorial clock auctions always lead to high efficiency? An experimental analysis of spectrum auction designs. Exp Econ 16(4):511-545

Bichler M, Hao Z, Adomavicius G (2017) Coalition-based pricing in ascending combinatorial auctions. Inf Syst Res 28(1):159-179

Brunner C, Goeree JK, Holt CA, Ledyard JO (2010) An experimental test of flexible combinatorial spectrum auction formats. Amer Econ J Microecon 2(1):39-57

Bykowsky MM, Cull RJ, Ledyard JO (2000) Mutually destructive bidding: the FCC auction design problem. J Regul Econ 17(3):205-228

Chernomaz K, Levin D (2012) Efficiency and synergy in a multi-unit auction with and without package bidding: an experimental study. Games Econ Behav 76(2):611-635

Cramton P (2007) How best to auction oil rights. In: Humphreys M, Sachs J, Stiglitz JE (eds) Escaping the resource curse, chap 5. Cambridge Univ Press, Cambridge, pp 114-151

Cramton P, Schwartz J (2000) Collusive bidding: lessons from the FCC spectrum auctions. J Regul Econ 17(3):229-252

Day RW, Raghavan S (2008) A combinatorial procurement auction featuring bundle price revelation without free-riding. Decis Support Syst 44(3):621-640

Fischbacher U (2007) z-tree: Zurich toolbox for ready-made economic experiments. Exp Econ 10(2):171-178

Goeree JK, Lien Y (2014) An equilibrium analysis of the simultaneous ascending auction. $\mathrm{J}$ Econ Theor 153:506-533
Goossens DR, Onderstal S, Pijnacker J, Spieksma FCR (2014) Solids: a combinatorial auction for real estate. Interfaces 44(4):351-363

Guler K, Bichler M, Petrakis I (2016) Ascending combinatorial auctions with risk averse bidders. Group Decis Negot 25(3):609-639

Ignatius J, Hosseini-Motlagh SM, Goh M, Sepehri MM, Mustafa A, Rahman A (2014) Multiobjective combinatorial auctions in transportation procurement. Math Probl Eng 2014:1-9

Kagel JH, Lien Y, Milgrom P (2010) Ascending prices and package bidding: a theoretical and experimental analysis. Am Econ $\mathrm{J}$ Microecon 2(3):160-85

Kazumori E (2010) Core-selecting auctions: An experimental study. CARF F-Series CARF-F-226, Center for Advanced Research in Finance, Faculty of Economics, The University of Tokyo

Nisan N (2000) Bidding and allocation in combinatorial auctions. In: Proceedings of the 2 nd acm conference on electronic commerce, EC '00, pp 1-12

Petrakis I, Ziegler G, Bichler M (2013) Ascending combinatorial auctions with allocation constraints: on game theoretical and computational properties of generic pricing rules. Inf Syst Res 24(3):768-786

Rassenti SJ, Smith VL, Bulfin RL (1982) A combinatorial mechanism for airport time slot allocation. Bell J Econ 13:402-417

Rothkopf MH, Pekeč A, Harstad RM (1998) Computationally manageable combinational auctions. Manag Sci 44(8):1131-1147

Sano R (2012) Non-bidding equilibrium in an ascending coreselecting auction. Games Econ Behav 74(2):637-650

Scheffel T, Pikovsky A, Bichler M, Guler K (2011) An experimental comparison of linear and nonlinear price combinatorial auctions. Inf Syst Res 22(2):346-368

Scheffel T, Ziegler G, Bichler M (2012) On the impact of package selection in combinatorial auctions: an experimental study in the context of spectrum auction design. Exp Econ 15(4):667-692

Vangerven B (2017) Combinatorial auctions: theory, experiments, and practice. $\mathrm{PhD}$ thesis, KU Leuven and Ghent University. https://biblio.ugent.be/publication/8541000. Accessed 25 Feb 2020

Vangerven B, Goossens DR, Spieksma FC (2017) Winner determination in geometrical combinatorial auctions. Eur $\mathrm{J}$ Oper Res 258(1):254-263 